\title{
(2) OPEN ACCESS \\ Measuring dynamic levels of self-perceived anxiety and concern during simulated mobility tasks in people with non-neovascular age-related macular degeneration
}

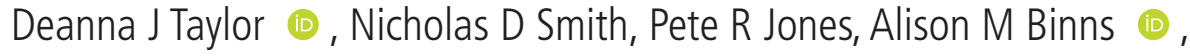 \\ David P Crabb
}

- Additional material is published online only. To view, please visit the journal online (http://dx.doi.org/10.1136/ bjophthalmol-2019-313864).

Division of Optometry and Visual Science, City, University of London, London, UK

\section{Correspondence to} Professor David P Crabb, Division of Optometry and Visual Science, City, University of London, London EC1V OHB, UK; David.Crabb.1@city.ac.uk

Received 8 January 2019 Revised 15 May 2019 Accepted 7 June 2019 Published Online First 9 July 2019

\begin{abstract}
Background/aims To assess response to real-world mobility scenarios in people with dry age-related macular degeneration (AMD) using a computer-based test. Methods Participants were shown 18 point-of-view computer-based movies simulating walking through real-world scenarios, and pressed a button during scenes which would cause them self-perceived anxiety or concern in their day-to-day life. Button pressure was recorded throughout. Pressure traces were generated, which aligned with each movie time point. Group averages based on AMD severity were generated. Bootstrapped confidence intervals (Cls) for responses by group were generated around traces. Traces were examined to discover events causing the greatest differences between groups.
\end{abstract}

Results Participants had early/no AMD $(n=8)$, intermediate AMD $(n=7)$ or geographic atrophy $(n=15$ (GA)). Median (IQR) logMAR visual acuity was 0.04 $(-0.04,0.18), 0.26(0.10,0.40)$ and $0.32(0.20,0.56)$, respectively. Participants with intermediate $A M D$ or $G A$ recorded greater pressure than those with early and no AMD (Kruskal-Wallis, $p=0.04$ ). Four events involving navigating stairs and three under low luminance elicited greatest differences between groups $(p<0.001)$.

Conclusion People with intermediate AMD or GA likely experience higher levels of concern associated with mobility. The test highlights areas of specific concern. Results should be useful in patient management and educating the public about the everyday effects of AMD.

\section{INTRODUCTION}

Mobility, specifically physical activity, such as walking, is crucial for well-being. Increased mobility is related to improved physical and mental health, ${ }^{1}{ }^{2}$ while decreased mobility is associated with poor health, social isolation and depression. ${ }^{3}$ Lack of mobility may result in frailty, contributing to a vicious cycle of ill-health and immobility. ${ }^{4}$ Agerelated macular degeneration (AMD) is known to impact negatively on mobility. A recent systematic review ${ }^{5}$ reported mobility as the most frequent subject of published studies on visual disability in AMD. However, only two studies ${ }^{67}$ focused specifically on people with non-neovascular (or dry) AMD, while one other ${ }^{8}$ considered AMD type in its analysis. Given the substantially higher prevalence of dry AMD as compared with neovascular AMD (nAMD), there is a clear gap in the literature on mobility in dry AMD.

One way of assessing physical mobility is through self-report. For example, self-report has been used to explore the effects of AMD on fall rates ${ }^{9}$ and fear of falling. ${ }^{10}$ Another approach involves mobility tasks in a lab-based setting, for example, navigating an obstacle course. ${ }^{11}$ In one study, eye movements and pupil diameter were measured while participants watched a movie of a journey while indicating sections of the journey deemed to be difficult. ${ }^{12}$ Another method of assessing mobility is in the 'real-world', for example, measuring road-crossing behaviour in the street $^{13}$ or via cellular tracking devices. ${ }^{14}$ While these studies provide useful information, one aspect of mobility that has had little attention in the AMD literature is anxiety and concern surrounding mobility. This may present a barrier to mobility tasks, resulting in consequences of decreased mobility described above.

The aim of this study was to assess distress associated with mobility situations in people with dry $\mathrm{AMD}$, in a safe environment simulating real-life mobility scenarios, using a novel computer-based test. In particular, we aimed to determine the effect of AMD severity, and to assess which types of mobility situations cause the greatest response among people with dry AMD. A secondary aim was to explore potential predictors of self-perceived concern/anxiety related to mobility.

\section{METHODS}

People with dry AMD were recruited from Moorfields Eye Hospital Trust, London, through optometrists local to the university and through the Macular Society (www.macularsociety.org). AMD participants were required to be aged $\geq 60$ years, have sufficiently clear ocular media (Lens Opacities Classification System (LOCS) III grade $<3$ ), adequate pupillary dilation and fixation to allow quality fundus imaging, and have dry AMD in their better-seeing eye. Fellow eyes could be of any AMD status. Binocular visual acuity (VA) was required to be $\log$ MAR 0.7 or better (Snellen equivalent of 6/30) using an Early Treatment Diabetic Retinopathy Study (ETDRS) chart. Exclusion criteria were nAMD in the better-seeing eye, ocular or systemic diseases that could affect visual function or history 


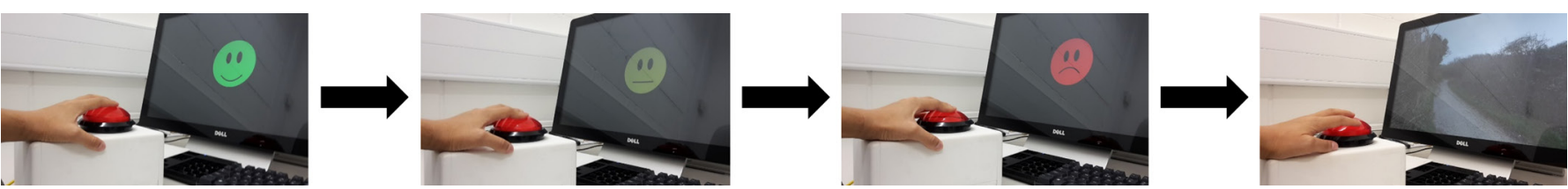

Figure 1 Photographs of big button test setup.

of medication known to affect macular function, or high risk of angle closure during pupillary dilation. Participants were required to pass an abridged version of the Mini-Mental State Evaluation $^{15}$ and have sufficient knowledge of the English language to understand the Participant Information Sheet, undergo history and symptoms questioning and understand instructions.

Visually healthy controls were recruited from the City Sight Optometry Clinic at City, University of London. Eligibility criteria were same as for people with AMD except participants were required to have no AMD (or other eye diseases) in either eye, and monocular VA of logMAR $0.3(6 / 12)$ or better in each eye.

The study was approved by Nottingham 2 National Health Service (NHS) Research Ethics Committee and was conducted according to the tenets of the Declaration of Helsinki. Written, informed consent was obtained from each participant prior to examination. Participant information was anonymised before being entered into a secure computer database.

Participants underwent baseline examinations to evaluate AMD status and ensure eligibility. Structured history and symptoms were taken including the EQ-5D questionnaire ${ }^{16}$ to assess health-related quality of life (HRQoL). Best corrected logMAR VA was determined with subjective refraction using an ETDRS chart. Contrast sensitivity (CS) was tested with a Pelli-Robson chart at 1 metre (binocularly) with best-corrected distance prescription and recorded as $\log C S$.

Following study tests, participants underwent dilated fundus examination. Lens clarity was graded (LOCS III grading scale). Digital colour fundus photographs were obtained and used to classify and grade AMD status by the better-seeing eye (determined by VA) as early, intermediate or late according to the Beckman classification scale. ${ }^{17}$ Spectral-domain optical coherence tomography (OCT) and fundus autofluorescence images were taken; these, along with slit lamp indirect ophthalmoscopy, were used to support results obtained using colour fundus

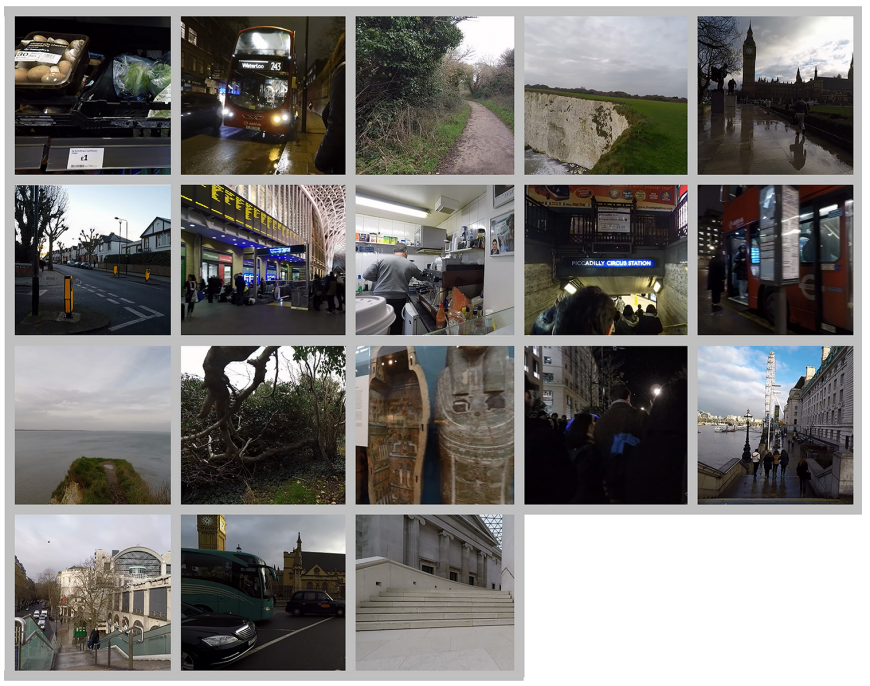

Figure 2 Still screenshots from each of the 18 movies. photographs, for example, OCT to confirm the presence of nAMD, or fundus autofluorescence to confirm the presence of GA.

Microperimetry was performed using the MAIA microperimeter (CenterVue, Padova, Italy). A total of 37 points were tested over the central $10^{\circ}$ of the retina, measured using white Goldmann III targets presented for $200 \mathrm{~ms}$, and thresholds were calculated using the system's full threshold 4-2 staircase strategy.

\section{Experimental procedure}

Participants were seated $50 \mathrm{~cm}$ away from a Dell 23-inch touch monitor-P2314T (resolution $1920 \times 1080$ ). Participants were tested binocularly wearing habitual intermediate correction. A box with a big button (BB) on the top was set in front of the monitor. This contained a custom-built force plate (capable of detectingcontinuous variations in pressure), which was interfaced using an Arduino Microcontroller (http://www. arduino.cc/). The test consisted of 18 short movies, shown in a randomised order. These were filmed from the point of view of someone walking through different real-world scenarios using a GoPro Hero4 camera mounted on a Feiyu Tech G4 3-Axis Handheld Steady Gimbal. The movie clips covered a range of walking settings, including light and dark conditions, indoor and outdoor, and rural and urban. Each of the movies showed situations which may induce anxiety for a person with visual impairment. Scenarios included walking up and down stairs, walking around a museum, crossing roads, handling money and walking around a supermarket. The content was chosen based on our previous systematic review ${ }^{5}$ and interviews with individuals with dry AMD.

Test duration was $30 \mathrm{~min}$ (movie clips lasted between 29 and $188 \mathrm{~s}$; average duration $80 \mathrm{~s}$ ). Participants were instructed to press down on the button with their dominant hand whenever they felt the footage to show a situation which would cause them anxiety or concern and to keep the button pressed until they felt that the situation had passed. Pressure on the BB was recorded throughout and quantified at each time point on a scale of 0 (no pressure) to -1 (fully pressed). A demonstration in which a cartoon face appeared to smile when the BB was unpressed, frown when the $\mathrm{BB}$ was fully pressed and appeared neutral when the $\mathrm{BB}$ was semi-pressed was shown at the start of the test and in between each of the trials. Participants were asked to press the button 10 times each time they viewed the demonstration screen. This was used to assess whether differences in mean pressure were apparent between groups while not watching a movie (due to muscle weakness or frailty). Setup of the experimental procedure with the demonstration screen and one of the test movies are shown in figure 1 and still screenshots from each of the 18 movies are shown in figure 2 .

\section{Data analysis}

Mean BB pressure across all movies was the primary outcome measure (BB response). Participants were grouped by AMD severity based on the Beckman classification ${ }^{17}$ in their betterseeing eye. The distribution of pressure data was skewed so 
Table 1 Summary of clinical measures expressed as median (IQR)

\begin{tabular}{|c|c|c|c|c|}
\hline & $\begin{array}{l}\text { Controls } \\
\text { (early and no AMD, n=8) }\end{array}$ & $\begin{array}{l}\text { Intermediate AMD } \\
(n=7)\end{array}$ & $\begin{array}{l}\mathrm{GA} \\
(\mathrm{n}=15)\end{array}$ & $P$ value \\
\hline Age, years & $77(72,80)$ & $82(70,86)$ & $75(70,78)$ & 0.20 \\
\hline Binocular VA & $0.04(-0.04,0.18)$ & $0.26(0.10,0.40)$ & $0.32(0.20,0.56)$ & 0.01 \\
\hline Better eye VA & $0.10(0.00,0.18)$ & $0.26(0.20,0.42)$ & $0.36(0.32,0.60)$ & $<0.01$ \\
\hline Worse eye VA & $0.28(0.06,0.42)$ & $0.58(0.26,1.58)$ & $0.86(0.40,1.02)$ & 0.01 \\
\hline CS & $1.95(1.65,1.95)$ & $1.60(1.35,1.65)$ & $1.60(1.25,1.65)$ & 0.01 \\
\hline Better eye AT & $27.8(26.1,29.8)$ & $26.5(25.8,27.8)$ & $23.4(19.0,25.6)$ & $<0.01$ \\
\hline Worse eye AT & $27.0(25.6,29.5)$ & $25.0(23.8,27.4)$ & $20.4(6.9,23.8)$ & $<0.01$ \\
\hline EQ-5D index score & $0.89(0.72,1.00)$ & $0.81(0.72,1.00)$ & $0.77(0.72,1.00)$ & 0.57 \\
\hline
\end{tabular}

$P$ values for between-group differences were calculated using Kruskal-Wallis tests.

AMD, age-related macular degeneration; AT, MAIA microperimetry average threshold; CS, Pelli-Robson contrast sensitivity (logCS);GA, geographic atrophy; VA, visual acuity $(\log M A R)$

Kruskal-Wallis tests were used to test for differences between groups. Relationship between BB response and HRQoL was assessed by grouping participants according to their EQ-5D responses and testing for differences between these groups. Univariate associations (Spearman's rho) between mean BB response and age, VA (better eye, worse eye and binocular), CS and average retinal sensitivity threshold (better eye and worse eye) as measured by microperimetry were explored.

The test generates a pressure trace that is aligned with time points throughout the movies to estimate the response at each moment. The trace can be derived such that it is an average from a group of participants. Bootstrapping was used to compute CIs for the meanresponse of each group around these traces ( $N=20$ 000; bias-corrected and accelerated method). Traces were examined to discover which types of mobility situations caused greatest differences between people with dry AMD and controls. Statistical analysis was carried out using SPSS Statistics V.22 and MATLAB R2016b (The Mathworks, Natick, Massachusetts, USA).

\section{RESULTS}

A total of 30 participants completed the BB test ( $n=5$ no AMD, $\mathrm{n}=3$ early $\mathrm{AMD}, \mathrm{n}=7$ intermediate $\mathrm{AMD}$ and $\mathrm{n}=15 \mathrm{GA}$ ) between March 2016 and March 2017. Visual function and demographic characteristics are presented in table 1. All visual function parameters differed significantly between groups based on AMD severity; age and EQ-5D index scores did not significantly differ ( $p>0.05$; Kruskal-Wallis test). There was no difference in BB response between groups when not watching a movie ( $\mathrm{p}>0.05$; Kruskal-Wallis test).

\section{Determining the effect of AMD severity on button response}

Median (IQR) averaged BB response across all movies were: no AMD $0.00(-0.03,0.0)$; early AMD $-0.03(-0.04,0)$; intermediate AMD $-0.08(-0.19,-0.02)$ and GA $-0.07(-0.17,-0.04)$. The average response for people with early AMD and no AMD differed significantly from those with intermediate AMD and GA (Kruskal-Wallis; $p=0.04$, figure 3). Median (IQR) collated $\mathrm{BB}$ response for people with intermediate AMD and GA was $-0.07(-0.17,-0.02)$ and median (IQR) BB response from people with no AMD or early AMD was $0.00(-0.04,0.00)$. If we consider the quartile of each distribution with the greatest $\mathrm{BB}$ response then the response was more than fourfold greater in people with intermediate AMD and GA compared with those with no AMD or early AMD.

\section{Which types of mobility situations cause the greatest button response?}

The pressure trace showing mean $\mathrm{BB}$ response at each time point across the movies for the four AMD classification groups is available in online supplementary file 1.

From our initial analysis, it was clear that people with no AMD and early AMD showed similar BB response, and that people with intermediate AMD and GA showed similar BB response. To simplify visualisation and analysis, we, therefore, generated traces grouping no AMD and early AMD together as 'controls', and intermediate AMD and GA as the 'AMD' group (figure 4). Inspection of this figure shows that within certain movies there were specific events where BB response for people with AMD and controls deviated significantly $(\mathrm{p}<0.001$; greyshaded regions of figure 4; see figure legend for details). Four of these events involved negotiating stairs; other events where these differences occurred involved avoiding cyclists on a rural path, flagging down a bus at night, navigating a crowd at night, crossing a road at night, reading a signpost with a map on it

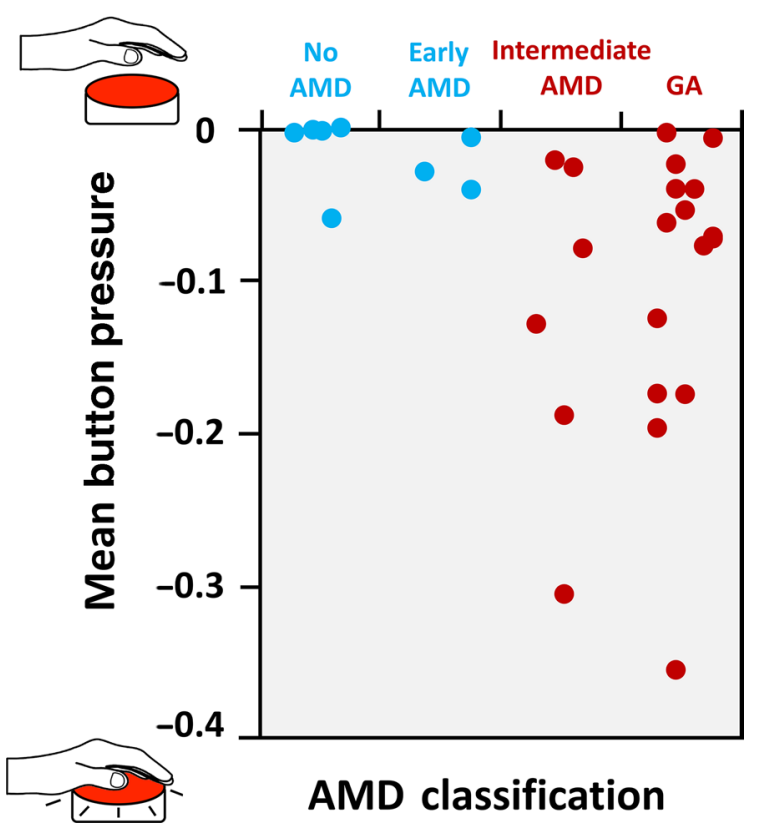

Figure 3 Mean big button response across all movies stratified by AMD classification (points have been horizontally jittered to avoid overlap). AMD, age-related macular degeneration; GA, geographic atrophy. 


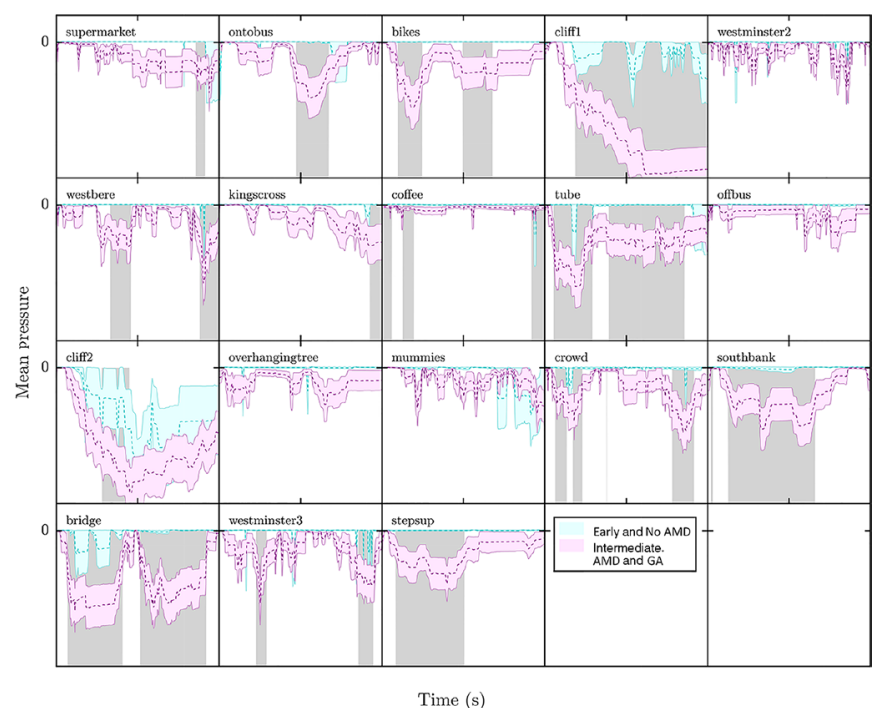

Figure 4 Mean (SE) BB response (dotted line=mean; solid lines=SE) for the entire duration of each of the 18 movies shown. Grey regions on chart indicate time periods at which the difference in $B B$ response between groups was significantly different $(p<0.001)$, that is, situations which caused particular anxiety to individuals with dry AMD compared with controls. AMD, age-related macular degeneration; BB, big button; GA, geographic atrophy.

and walking along a cliff path with a steep drop on one side. Figure 5 shows example still shots from one movie with the trace shown; example movies can be viewed in online supplementary files 2-4.

\section{Exploring potential predictors of self-perceived concern or anxiety related to mobility}

No significant correlations were found between BB response and: VA (binocular, better eye and worse eye), binocular CS, MAIA average threshold (better eye and worse eye) or EQ-5D index score (Spearman's rho; $\mathrm{p}>0.05$ ). Scatter plots showing these relationships can be viewed in online supplementary file 5. There was no difference in BB response between people who reported 'no difficulty' with mobility on EQ-5D $(n=21)$ to those who reported 'some difficulty' $(n=9)$ (Mann-Whitney; $p>0.05)$. No participants reported extreme difficulty with mobility on EQ-5D.

\section{DISCUSSION}

It is well documented that people with AMD have difficulties with mobility. This study adds to this by exploring the effect dry AMD severity has on self-perceived mobility anxiety, and assessing predictors of this. We evaluated which types of mobility situations cause the greatest self-perceived anxiety for people with dry AMD. Our methods were novel and offer an important advantage over methods of assessing mobility directly in the real world or in a built life-like environment, such as the Pedestrian Accessibility and Movement Environment Laboratory at University College London, $\mathrm{UK}^{18}{ }^{18}$ and the Streetlab artificial street, Paris, France, ${ }^{19}$ in that they may be conducted in a safe environment, free from health and safety risks. The test may be presented in different ways, including through virtual/ augmented reality platforms, and on portable electronic devices. It also allows for the simulation of a variety of several different environments, that is, indoor/outdoor, rural/urban, and so on.

Our results suggest that individuals with intermediate and late dry AMD are more likely to experience self-perceived response anxiety to mobility situations than individuals with early AMD and those without AMD. Notably, we report increased perceived anxiety related to mobility in people with intermediate AMD (ie, large drusen, with or without pigmentary changes). This group is often considered to be affected minimally by AMD. For example, over half would meet UK vision standards for driving, and would certainly not be considered 'sight-impaired'.

The greatest differences in BB response between groups was observed during scenes showing negotiating stairs, map reading, as well as walking along a cliff edge. Participants with intermediate and late dry AMD consistently exhibited higher levels of self-perceived mobility anxiety during scenes showing stair negotiation, suggesting this is a particularly troublesome task. This worry is not unfounded; a literature review of stair negotiation in ageing ${ }^{20}$ reported falls on stairs to be a leading cause of accidental death, that stair injuries increased with age and that perceived difficulty with stair negotiation is frequently reported among older people. These difficulties are likely exacerbated in AMD and several studies report alterations to movement and gait characteristics in AMD in an attempt (consciously or subconsciously) to counteract these difficulties. ${ }^{21} 22$ Three situations which caused a large difference in BB response between groups related to vision under low luminance. This supports the literature suggesting scotopic sensitivity loss and problems in low luminance conditions may precede other functional loss in $\mathrm{AMD}^{23}$

Our results were not directly related to clinical measures of visual function (VA, CS and microperimetry). These results support those of Donoghue et $a l,{ }^{24}$ who suggest that VA and CS, while contributing to mobility performance, do not contribute to fear of falling in low vision. Other measures of visual function that were not assessed may be better predictors of perceived mobility response anxiety. This is a limitation of our study. For example, earlier research has highlighted the importance of the photostress test and cone threshold, ${ }^{25}$ binocular central scotoma size, ${ }^{11}$ scanning ability ${ }^{26}$ and differential velocity threshold ${ }^{6} 26$ in predicting mobility performance. One study ${ }^{27}$ reported the best predictor for self-reported mobility among people with low
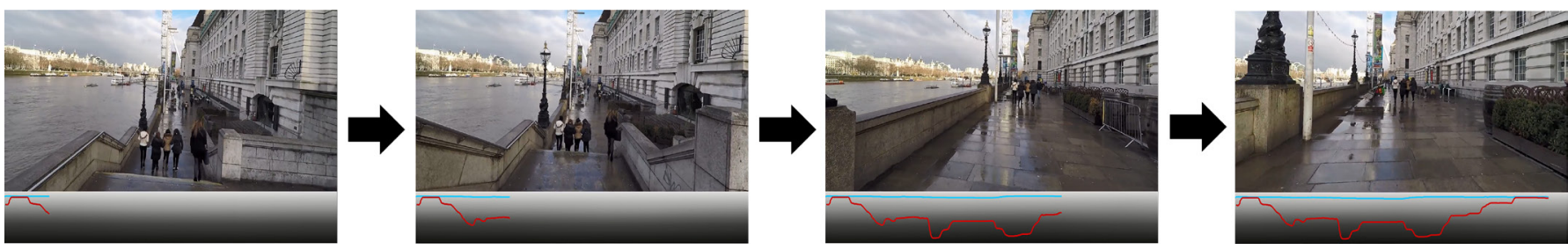

Figure 5 Example still shots from the movie 'southbank' with the corresponding pressure trace. The blue trace represents button pressure from people with no AMD and early AMD, and the red trace represents button pressure from people with intermediate AMD and GA. Example movies can be found in online supplementary files 2-4. AMD, age-related macular degeneration; GA, geographic atrophy. 
vision to be binocular visual field, followed by scanning ability, low-contrast VA and finally, high-contrast VA; while another ${ }^{28}$ reported reduced VA and CS to predict mobility restriction in AMD.

No relationship was found between HRQoL and BB response. We examined responses specifically to the EQ-5D item related to mobility. It was unexpected that people who reported 'no difficulty' with mobility did not differ in their BB response, on average, from those who reported 'some difficulty' with mobility. However, the EQ-5D item measures self-reported mobility difficulty, whereas the BB test measures self-reported mobility anxiety within specific situations. It is also possible that a person may report no difficulties with mobility in their dayto-day life because they have self-imposed mobility restrictions (either consciously or subconsciously), which lead to avoidance of anxiety-inducing situations. Previous research has suggested that people with vision loss from AMD do limit activities and mobility. ${ }^{3} 9$ The BB test may, therefore, show situations that these participants would not consider when rating the mobility difficulty in their day-to-day lives as these situations would normally be avoided. We did not ask participants about the previous history of falls; this might increase anxiety levels associated with mobility and we hope that future work will investigate this.

This study has advanced our understanding of the relationship between AMD and mobility-related anxiety. However, there are limitations which must be acknowledged. First, it would be impossible to capture all possible real-world mobility scenarios that a person with AMD might encounter. Therefore, we have captured a variety of situations, including daytime and nighttime, urban and rural, and indoor and outdoor, in order to assess an array of potential scenarios, while keeping duration brief enough to maintain participants' attention and limit fatigue. A second limitation relates to our setting. All participants were volunteers who had sufficient confidence in their own mobility to travel to City, University of London to participate. We, therefore, are likely to be underestimating the magnitude of this problem for the wider population of people with dry AMD. Future work might take a version of this test into communities in order to capture a wider population. Anxiety levels could be measured physiologically in real-time indirectly by using skin conductance and variations in heart or breathing rate. Skin conductance has been used in previous ophthalmic research to assess anxiety during eye examinations and contact lens fitting. ${ }^{3031}$ This would have been beyond the scope of this study, which focused on individuals' perceived anxiety in response to mobility situations, but future work could compare results from the $\mathrm{BB}$ test with measurements of anxiety using this method. Further investigation might link results to personality type ${ }^{30}$ and state and trait anxiety. ${ }^{31}$ There is some debate in the literature as to which type of visual field loss is more detrimental to mobility. Some studies (eg, Subhi $e t \mathrm{al}^{32}$ ) report peripheral field loss to cause greater mobility problems, while others (eg, Turano et $a l^{33}$ ) report central visual field loss to be more problematic for mobility. This question could be addressed by replicating this study in people with peripheral visual field loss for comparison. Finally, the results presented here are limited by our sample size; future work ought to test this in larger numbers.

In summary, our test is a useful measure of self-perceived mobility anxiety for people with dry AMD. People with intermediate and late dry AMD are likely to experience higher levels of perceived concern or anxiety during everyday mobility tasks than people with early and without AMD. The relationship between mobility anxiety and other variables, such as visual function and psychological factors, is likely complex and requires further study. The BB test highlights areas of specific concern to people with dry AMD; negotiating stairs and vision under low luminance emerged as particularly troublesome. The test has the potential to be used in other eye diseases, and as a patient-centred outcome for interventions relating to mobility. The results have the potential to be useful in both patient management and educating members of the public about the real-world effects of AMD.

Acknowledgements The authors thank the Macular Society for their invaluable help with participant recruitment.

Contributors DJT: study design, data collection, data analysis and manuscript preparation. NDS: study design. PRJ: data analysis and manuscript critique. DPC and $A B$ : study conception and design, data analysis and manuscript critique.

Funding This study was funded as part of an unrestricted investigator-initiated research grant from Roche Products Ltd, UK, awarded to DPC.

Disclaimer The sponsor or funding organization had no role in the design or conduct of this research.

Competing interests None declared.

Patient consent for publication Not required.

Ethics approval The study was approved by Nottingham 2 National Health Service (NHS) Research Ethics Committee, and was conducted according to the tenets of the Declaration of Helsinki

Provenance and peer review Not commissioned; externally peer reviewed.

Data availability statement Data are available on request.

Open access This is an open access article distributed in accordance with the Creative Commons Attribution Non Commercial (CC BY-NC 4.0) license, which permits others to distribute, remix, adapt, build upon this work non-commercially, and license their derivative works on different terms, provided the original work is properly cited, appropriate credit is given, any changes made indicated, and the use is non-commercial. See: http://creativecommons.org/licenses/by-nc/4.0/.

\section{ORCID iDs}

Deanna J Taylor http://orcid.org/0000-0001-8261-5225

Alison M Binns http://orcid.org/0000-0001-8621-498X

\section{REFERENCES}

1 Collins AL, Goldman N, Rodríguez G. Is positive well-being protective of mobility limitations among older adults? J Gerontol B Psychol Sci Soc Sci 2008:63:P321-P327.

2 Shimada H, Ishizaki T, Kato M, et al. How often and how far do frail elderly people need to go outdoors to maintain functional capacity? Arch Gerontol Geriatr 2010;50:140-6.

3 Wang MY, Rousseau J, Boisjoly $\mathrm{H}$, et al. Activity limitation due to A fear of falling in older adults with eye disease. Invest Ophthalmol Vis Sci 2012;53:7967-72.

4 Xue Q-L, Fried LP, Glass TA, et al. Life-space constriction, development of frailty, and the competing risk of mortality: the women's health and aging study I. Am J Epidemiol 2008;167:240-8.

5 Taylor DJ, Hobby AE, Binns AM, et al. How does age-related macular degeneration affect real-world visual ability and quality of life? A systematic review. BMJ Open 2016:6:e011504.

6 Brown B, Brabyn L, Welch $L$, et al. Contribution of vision variables to mobility in agerelated maculopathy patients. Optometry and Vision Science 1986;63:733-9.

7 Lovie-Kitchin JE, Brown B. Reaction time in age-related maculopathy. Am J Optom Physiol Opt 1986;63:366-71.

8 Sengupta S, van Landingham SW, Solomon SD, et al. Driving habits in older patients with central vision loss. Ophthalmology 2014;121:727-32.

9 Szabo SM, Janssen PA, Khan K, et al. Neovascular AMD: an overlooked risk factor for injurious falls. Osteoporos Int 2010:21:855-62.

10 Popescu ML, Boisjoly H, Schmaltz H, et al. Explaining the relationship between three eye diseases and depressive symptoms in older adults. Invest Ophthalmol Vis Sci 2012;53:2308-13.

11 Hassan SE, Lovie-Kitchin JE, Woods RL. Vision and mobility performance of subjects with age-related macular degeneration. Optom Vis Sci 2002;79:697-707.

12 Aspinall PA, Borooah S, Al Alouch C, et al. Gaze and pupil changes during navigation in age-related macular degeneration. Br J Ophthalmol 2014;98:1393-7.

13 Geruschat DR, Hassan SE, Turano KA, et al. Gaze behavior of the visually impaired during street crossing. Optom Vis Sci 2006;83:550-8.

14 Curriero FC, Pinchoff J, van Landingham SW, et al. Alteration of travel patterns with vision loss from glaucoma and macular degeneration. JAMA Ophthalmol 2013;131:1420-6. 
15 Folstein MF, Folstein SE, McHugh PR. "Mini-mental state". A practical method for grading the cognitive state of patients for the clinician. J Psychiatr Res 1975;12:189-98.

16 Rabin R, de Charro F, Charro Fde. EQ-5D: a measure of health status from the EuroQol group. Ann Med 2001;33:337-43.

17 Ferris FL, Wilkinson CP, Bird A, et al. Clinical classification of age-related macular degeneration. Ophthalmology 2013;120:844-51.

18 McCarthy I, Suzuki T, Tyler N, et al. Mobility in the built environment: age-related changes in gait characteristics when walking on complex terrain. Healthy Aging Res 2016:5:1-9.

19 Authié CN, Berthoz A, Sahel J-A, et al. Adaptive gaze strategies for locomotion with constricted visual field. Front Hum Neurosci 2017;11.

20 Startzell JK, Owens DA, Mulfinger LM, et al. Stair negotiation in older people: a review. J Am Geriatr Soc 2000;48:567-80.

21 Kotecha A, Chopra R, Fahy RTA, et al. Dual tasking and balance in those with central and peripheral vision loss. Invest Ophthalmol Vis Sci 2013;54:5408-15.

22 Wood JM, Lacherez PF, Black AA, et al. Postural stability and gait among older adults with age-related maculopathy. Invest Ophthalmol Vis Sci 2009;50:482-7.

23 Owsley C, McGwin G, Scilley K, et al. Development of a questionnaire to assess vision problems under low luminance in age-related maculopathy. Invest Ophthalmol Vis Sci 2006;47:528-35.
24 Donoghue OA, Ryan H, Duggan E, et al. Relationship between fear of falling and mobility varies with visual function among older adults. Geriatr Gerontol Int 2014; 14:827-36.

25 Mathew RSet al. Predictors of poor mobility in people with age-related macular degeneration. Invest Ophthalmol Vis Sci 2008;49.

26 Kuyk T, Elliott JL, Fuhr PS. Visual correlates of obstacle avoidance in adults with low vision. Optom Vis Sci 1998;75:174-82.

27 Bibby SA, Maslin ER, Mcllraith R, et al. Vision and self-reported mobility performance in patients with low vision. Clin Exp Optom 2007;90:115-23.

28 Sengupta S, Nguyen AM, van Landingham SW, et al. Evaluation of real-world mobility in age-related macular degeneration. BMC Ophthalmology 2015;15.

29 Popescu ML, Boisjoly H, Schmaltz H, et al. Age-related eye disease and mobility limitations in older adults. Invest Ophthalmol Vis Sci 2011;52:7168-74.

30 Denollet J. DS14: standard assessment of negative affectivity, social inhibition, and type D personality. Psychosom Med 2005;67:89-97.

31 Spielberger CDet al. Manual for the state-trait anxiety inventory. Palo Alto, CA: Consulting Psychologists Press, 1983

32 Subhi H, Latham K, Myint J, et al. Functional visual fields: relationship of visual field areas to self-reported function. Ophthalmic Physiol Opt 2017;37:399-408.

33 Turano KA, Broman AT, Bandeen-Roche K, et al. Association of visual field loss and mobility performance in older adults: Salisbury Eye Evaluation study. Optom Vis Sci 2004;81:298-307. 